\title{
Axion induced spin effective couplings
}

\author{
Zihang Wang $\oplus^{1,2, *}$ and Lijing Shao $\odot^{2,3}$ \\ ${ }^{1}$ Department of Astronomy, School of Physics, Peking University, Beijing 100871, China \\ ${ }^{2}$ Kavli Institute for Astronomy and Astrophysics, Peking University, Beijing 100871, China \\ ${ }^{3}$ National Astronomical Observatories, Chinese Academy of Sciences, Beijing 100012, China
}

(Received 14 February 2021; accepted 1 June 2021; published 24 June 2021)

\begin{abstract}
Detecting axionic dark matter induced electron or nucleon oscillating electric dipole moment (OEDM) has become a new way for dark matter searches. We reexamine such axion-spin couplings in external electromagnetic fields. We point out that axion-photon interaction induces an electron spin effective coupling, which is different from an OEDM. In particular, the axion-spin effective coupling is directly related to magnetic field rather than electric field. For axion-electron or axion-nucleon couplings, an OEDM of fermion is introduced, whose effect in ultralight axion cases depends on whether axion shift symmetry is manifest. Specifically, ultralight axionic dark matter interactions that do not obey the shift symmetry will be strongly constrained. We also extend the results to the case where axion has a finite velocity.
\end{abstract}

DOI: 10.1103/PhysRevD.103.116021

\section{INTRODUCTION}

It has been known from various observations that most of the substance in the Universe is invisible, in the form of dark matter and dark energy [1,2]. We observe dark matter only through gravitational interaction, but we have never detected them directly yet. Weakly interacting massive particles (WIMPs) [3] are important candidates for dark matter. Many proposed dark matter direct detection experiments focus on detection of WIMPs, but after decades of efforts we still have not found any convincing signal yet [4]. Thus other kinds of dark matter candidates are getting more attention in recent years.

Another good dark matter candidate is the QCD axion, which was originally proposed to solve the strong $C P$ problem [5,6]. These theories introduce a global U(1) symmetry, the so-called the Peccei-Quinn symmetry. The symmetry is spontaneously broken below an energy scale $f_{a}$, and the axion is the resulting pseudo-Goldstone boson. From the chiral perturbation theory, the QCD axion parameters have a relation [7],

$$
m_{a}=5.70 \mu \mathrm{eV}\left(\frac{10^{12} \mathrm{GeV}}{f_{a}}\right)
$$

\footnotetext{
*wzhax@pku.edu.cn
}

Published by the American Physical Society under the terms of the Creative Commons Attribution 4.0 International license. Further distribution of this work must maintain attribution to the author(s) and the published article's title, journal citation, and DOI. Funded by SCOAP ${ }^{3}$. where $m_{a}$ is the axion mass. The relation holds for the QCD axion. In the string theory [8] or other beyond standard model theories, axionlike particles (ALPs) appear, whose properties are similar to the QCD axion but do not necessarily satisfy Eq. (1). ALPs can have a mass ranging from $\sim 10^{-33} \mathrm{eV}$ to $\mathrm{keV}$ scale or even larger. Depending on the mass, these ALPs play very different roles in astrophysics [9]. For example, an ALP with a mass $10^{-33} \mathrm{eV}$ is a candidate of the dark energy [10]. For an ALP with a mass around $10^{-22} \mathrm{eV}$, it is a candidate of the fuzzy dark matter [11]. For an ALP with a mass smaller than $10^{-10} \mathrm{eV}$, it may help explain the possible $\mathrm{TeV}$ transparency of the Universe [12]. The QCD axions with a mass around $10^{-5} \mathrm{eV}$ to $10^{-3} \mathrm{eV}$ are candidates of the cold dark matter [13-15] and may contribute to stellar cooling [16].

Ultralight axions refer to a type of ALPs with mass smaller than about $10^{-18} \mathrm{eV}$ [9]. In literature, ultralight axions have a mass around $10^{-22} \mathrm{eV}$ are often discussed [11], whose de Broglie wavelength is about the size of a galaxy. The wavelike behavior of ultralight axions may potentially solve the small scale crisis faced by cold dark matter models (e.g., the core-cusp problem and missingsatellite problem) [17].

Numerous methods have been proposed to probe axions or axionic dark matter, including the microwave cavity experiments like ADMX [18,19], "light shining through wall" experiments such as OSQAR [20], and solar axion observations like CAST [21]. Although these experiments have not found signal of axions, they have set constraints on axion interactions. Recently XENON1T experiments [22] detected a possible signal of solar axions, but the results are still controversial. 
Recently there are some new ideas of axionic dark matter detection. In particular, searches for axion induced oscillating electric dipole moment (OEDM) and axion induced spin precession become new ways for dark matter searches [23]. CASPEr [24] is a proposed experiment to detect nucleon OEDM using nuclear magnetic resonance. Detection of nucleon OEDM using storage ring methods has also been proposed [25]. Hill [26-29] proposed that axion-photon interaction also introduces an OEDM of the electron, and the electric dipole radiation induced by axionic dark matter is also discussed. Several experiments have been proposed to detect the OEDM of the electron [30,31]. Similarly, axion-electron interaction may introduce an electron OEDM and may be detected via spin precession [32]. Axion induced effects in atoms and molecules are discussed in Refs. [33,34]. Besides, many techniques $[35,36]$ in constraining electron static electric dipole moment (EDM) are helpful in axion detection as well. Thus studying axion-spin interaction is crucial and timely. Besides, there are extensions of these phenomena to macroscopic spin interactions. For example, effects of spin-axion coupling in curved spacetime that do not include electromagnetic fields are considered in Ref. [37]. Axion couplings may also introduce spin-dependent forces and may be detectable [38].

In this paper, we will reconsider axion-spin couplings in applied electric or magnetic field and find the effective interaction in nonrelativistic limit. We use the name "axion" but refer to general ALPs. We argue that axionphoton interaction introduces an electron spin effective coupling, which is not the same as OEDM. In particular, no physical effect occurs if a constant electric field is applied, which agrees with the results in Ref. [27]. We show that the spin effective interaction (for axions with a zero velocity) is not directly related to the electric field but related to the magnetic field. Because a time-varying electric field is always accompanied with a magnetic field, a time-varying electric field contributes to the spin effective interaction. A direct consequence of these results is that experiments in Refs. [30,31] can not be used to detect axion-photon interaction in the zero axion velocity case. Later in the paper we come to other axion interactions and include corrections from a finite axion velocity. We will stress the importance of the axion shift symmetry. For a type of ALP interaction that does not satisfy the shift symmetry, strong constraints for ultralight axion interactions are easily set by current experiments like static EDM measurements. For those axion interactions that satisfy the axion shift symmetry with only derivative axion couplings, the constraints from experiments are much weaker in the ultralight axion case. The effective spin interactions induced by such ultralight axion couplings are suppressed by at least a factor of $m_{a} L \ll 1$. As long as the characteristic size of experiments, $L$, is much smaller than the Compton wavelength of axions, these effects will be suppressed.
The paper is arranged as follows. In Sec. II, we will discuss the axion-photon interaction and its induced electron spin interactions. We will stress its difference with an electron OEDM. In fact, the induced electron spin interactions depend on magnetic field rather than electric field. Hence experiments trying to detect such effects must be carefully designed. Then we include axion velocity corrections. We will also extend the result to the case with a time-varying external field. In Sec. III, we will consider the axion-spin effective coupling induced by the axion-electron interaction. We will consider two types of effective axionelectron interactions found in literature, which turn out to give different results. In Sec. IV, we consider effects of axion-neutron interaction, which has been discussed in Ref. [23]. We compare it with the axion-electron interaction case. Finally, we come to an extended discussion in Sec. V. In this paper, Greek indices take values in $\{0,1,2,3\}$ while Latin indices take values in $\{1,2,3\}$.

\section{EFFECTS OF AXION-PHOTON INTERACTION}

In the presence of axionic dark matter, the electron spin interacts differently with the external electric or magnetic field, which may manifest through OEDM or spin precession effects. We will first consider the effective interaction induced by the axion-photon interaction. We will neglect axion velocity and assume a static external electric and magnetic field first in Sec. II A. Then in Sec. II B we extend the result to nonzero axion velocity case. We will consider time-varying electromagnetic field in Sec. II C.

Axion-photon interaction can be written as,

$$
\mathcal{L}_{a \gamma \gamma}=\frac{1}{4} g_{a \gamma \gamma} a F_{\mu \nu} \tilde{F}^{\mu \nu}
$$

where $a$ is the axion field, $g_{a y \gamma}$ is the axion-photon coupling constant, $F_{\mu \nu}=\partial_{\mu} A_{\nu}-\partial_{\nu} A_{\mu}$ is the electromagnetic field tensor, and its dual $\tilde{F}_{\mu \nu}$ is defined as,

$$
\tilde{F}_{\mu \nu}=\frac{1}{2} \epsilon_{\mu \nu \rho \sigma} F^{\rho \sigma} .
$$

Here we notice an important property,

$$
F_{\mu \nu} \tilde{F}^{\mu \nu}=\partial_{\mu}\left(2 \epsilon^{\mu \nu \rho \sigma} A_{\nu} \partial_{\rho} A_{\sigma}\right) .
$$

When we integrate by parts in action, we obtain,

$$
S_{\mathrm{int}}=-\int d^{4} x \frac{1}{2} g_{a \gamma \gamma}\left(\partial_{\mu} a(x)\right) \epsilon^{\mu \nu \rho \sigma} A_{\nu}(x) \partial_{\rho} A_{\sigma}(x) .
$$

The action above (except the axion mass term) satisfies a shift symmetry $a \rightarrow a+a_{0}$ where $a_{0}$ is constant. As we will see, axion induced spin couplings will be suppressed for small axion mass if axion interactions obey the shift symmetry. 


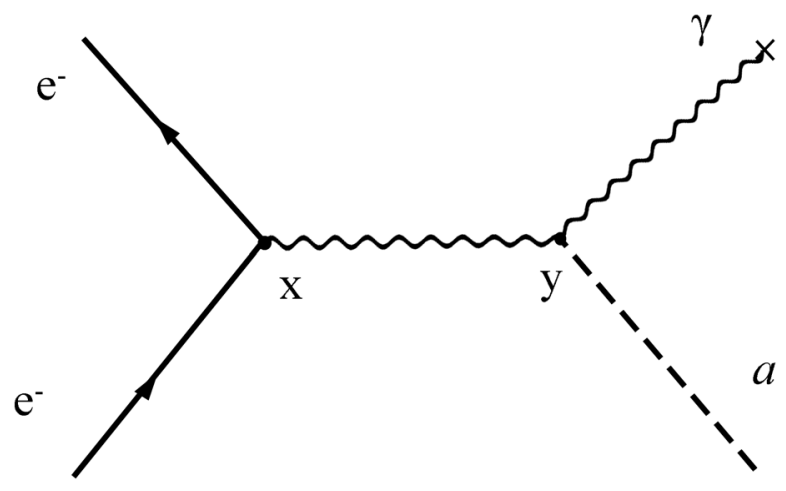

FIG. 1. Feynman diagram that contributes to electron spin effective interactions, which is induced by axion-photon interaction. In the diagram, $a$ is the axion field, $x$ and $y$ are interaction points. The cross on the right represents interaction with external electromagnetic fields.

\section{A. Axions with zero velocity}

It is well known that electron has a spin magnetic moment $\mu_{B}$, thus electron spin interacts with external magnetic field. As we will see below, due to the axionphoton interaction, the effective magnetic field seen from the electron is slightly modified. Hence the presence of axionic dark matter introduces an additional effective spin interactions.

The main contribution comes from the Feynman diagram shown in Fig. 1. We use the axion-photon interaction vertex

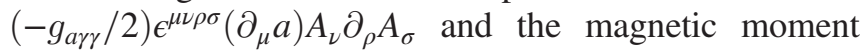
interaction $\left(-e /\left(2 m_{e}\right)\right) \bar{\psi} \sigma^{\mu \nu} \psi \partial_{\mu} A_{\nu}$. We use the magnetic moment interaction because it is much easier for us to take the nonrelativistic limit. The result is the same as the usual $-e \bar{\psi} \gamma \gamma^{\mu} \psi A_{\mu}$ interaction if we only consider spin interactions of the electron. In fact, the axion-spin effective couplings introduced by axion-photon interaction are only related to the magnetic moment of the particle. We will calculate the amplitude in coordinate space because it is easier to deal with the external field $A_{\mu}(x)$. The amplitude is,

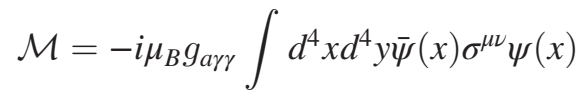

$$
\begin{aligned}
& \times\left(\frac{\partial}{\partial x^{\mu}} G(x-y)\right) \epsilon_{\lambda \nu \rho \eta} \partial^{\lambda} a(y) \partial^{\rho} A^{\eta}(y),
\end{aligned}
$$

where $\mu_{B}=e /\left(2 m_{e}\right)$ is the Bohr magneton. We also define,

$$
G(x-y)=-\int d^{4} q \frac{1}{q^{2}+i \epsilon} e^{i q \cdot(x-y)}
$$

We then consider the nonrelativistic limit of the electron,

$$
\psi(x)=\left(\begin{array}{c}
\chi(x) \\
\varphi(x)
\end{array}\right) e^{-i m_{e} x^{0}}
$$

In the nonrelativistic limit,

$$
\begin{gathered}
\bar{\psi}(x) \sigma^{i j} \psi(x) \rightarrow \chi^{\dagger}(x) \epsilon^{i j k} \sigma^{k} \chi(x), \\
\bar{\psi}(x) \sigma^{0 i} \psi(x) \rightarrow 0 .
\end{gathered}
$$

Hence the amplitude becomes,

$$
\begin{aligned}
\mathcal{M}= & -i \mu_{B} g_{a \gamma \gamma} \int d^{4} x d^{4} y \psi^{\dagger}(x) \sigma^{k} \psi(x) G(x-y) \\
& \times\left[\partial_{j}\left(\partial_{0} a(y) F^{k j}(y)\right)-\partial_{i}\left(\partial^{i} a(y) E^{k}(y)\right.\right. \\
& \left.\left.-\partial^{k} a(y) E^{i}(y)\right)\right]
\end{aligned}
$$

where the electric field $E^{k}=F^{k 0}$. We first consider the case that axionic dark matter has velocity $v=0$, hence $\partial_{i} a=0$. In the next subsection, we will come to velocity correction, which is suppressed by a factor $v / c \sim 10^{-3}$. The only term that remains is,

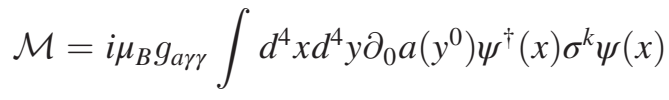

$$
\begin{aligned}
& \times G(x-y)(\nabla \times \vec{B})^{k}(y),
\end{aligned}
$$

In fact, $G(x-y)$ has a simple form in coordinate space. Equation (7) can be integrated directly. Define $t_{0}=x^{0}-y^{0}$, $r=|\vec{x}-\vec{y}|$, and we obtain,

$$
G(x-y)=-\frac{i}{4 \pi^{2}} \frac{1}{t_{0}^{2}-r^{2}-i \epsilon},
$$

where $\epsilon$ is a small positive number. We use $\frac{1}{x-i \epsilon}=\mathcal{P} \frac{1}{x}+$ $i \pi \delta(x)$ to obtain,

$$
G(x-y)=\frac{1}{4 \pi} \delta\left(t_{0}^{2}-r^{2}\right)-\frac{i}{4 \pi^{2}} \mathcal{P} \frac{1}{t_{0}^{2}-r^{2}},
$$

where $\mathcal{P} \frac{1}{x}$ can be defined as $\mathcal{P} \frac{1}{x}=\frac{x}{x^{2}+\epsilon^{2}}$. We also use,

$$
\delta\left(t_{0}^{2}-r^{2}\right)=\frac{1}{2 r}\left[\delta\left(t_{0}-r\right)+\delta\left(t_{0}+r\right)\right] .
$$

Now we can integrate Eq. (12) directly. We assume a coherent axion background field $a\left(y^{0}\right)=a_{0} e^{-i m_{a} y^{0}}$. We also use the result,

$$
\int d y^{0} \mathcal{P} \frac{e^{-i m_{a} y^{0}}}{y^{0}}=-\pi i
$$

For static fields, $\nabla \times \vec{B}=\vec{J}$, where $\vec{J}$ is the current density. The total contribution is,

\footnotetext{
${ }^{1}$ Note that the axion field is actually the real part of $a\left(y^{0}\right)$.
} 


$$
\begin{aligned}
\mathcal{M}= & \frac{i \mu_{B} g_{a \gamma \gamma}}{4 \pi} \int d^{4} x\left(\partial_{0} a\left(x^{0}\right)\right) \\
& \times \psi^{\dagger}(x) \sigma^{k} \psi(x) \int d^{3} y e^{i m_{a} r} \frac{J^{k}(\vec{y})}{r} .
\end{aligned}
$$

This is equivalent to an amplitude under an effective interaction,

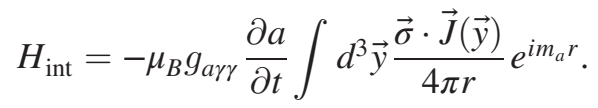

We can understand the axion-spin coupling induced by axion-photon interaction as follows. If axionic dark matter exists, in the presence of an external static magnetic field $\vec{B}_{0}$, an effective electromagnetic field will be induced. Assume that $\vec{B}_{0}$ is produced by a constant electric current $\vec{J}(\vec{x})$ and the axion has zero velocity. The induced electric field and magnetic field can be calculated from a perturbative calculation of field equations [39,40],

$$
\begin{gathered}
\vec{E}_{\mathrm{eff}}(\vec{x}, t)=-\frac{g_{a \gamma \gamma} a(t)}{4 \pi} \int d^{3} \vec{y} \frac{e^{i m_{a} r}-1}{r} \nabla \times \vec{J}(\vec{y}), \\
\vec{B}_{\mathrm{eff}}(\vec{x}, t)=\frac{i g_{a \gamma \gamma} m_{a} a(t)}{4 \pi} \int d^{3} \vec{y} \frac{e^{i m_{a} r}}{r} \vec{J}(\vec{y}),
\end{gathered}
$$

where we have used $a(t)=a_{0} e^{-i m_{a} t}$. We find that the effective Hamiltonian (18) is,

$$
H_{\text {int }}=-\mu_{B} \vec{\sigma} \cdot \vec{B}_{\text {eff }} .
$$

This can be interpreted as electron magnetic moment interacting with the effective magnetic field. In the limit $m_{a} r \ll 1, \vec{B}_{\text {eff }}$ becomes,

$$
\begin{aligned}
\vec{B}_{\mathrm{eff}}(\vec{x}, t) & =\frac{i g_{a \gamma \gamma} m_{a} a(t)}{4 \pi} \int d^{3} \vec{y} \frac{\vec{J}(\vec{y})}{r} \\
& =i g_{a \gamma \gamma} m_{a} a(t) \vec{A}(\vec{x}) .
\end{aligned}
$$

We note that in the limit $m_{a} r \ll 1$, which is true in experiments that search for ultralight axions, the effective Hamiltonian is proportional to the magnetic vector potential,

$$
\vec{A}(\vec{x})=\int d^{3} \vec{y} \frac{\vec{J}(\vec{y})}{4 \pi r} .
$$

As an illustration of the effective interaction, consider an electron (or a molecule in actual experiments) placed in the center of a cylinder shaped conductor, as shown in Fig. 2. The conductor is hollow. An electric current $I$ flows on the surface of the cylinder in $+z$ direction. The length of the conductor is $L$ and the radius of the cylinder is $R$. If there is no axionic dark matter present, the magnetic field in the

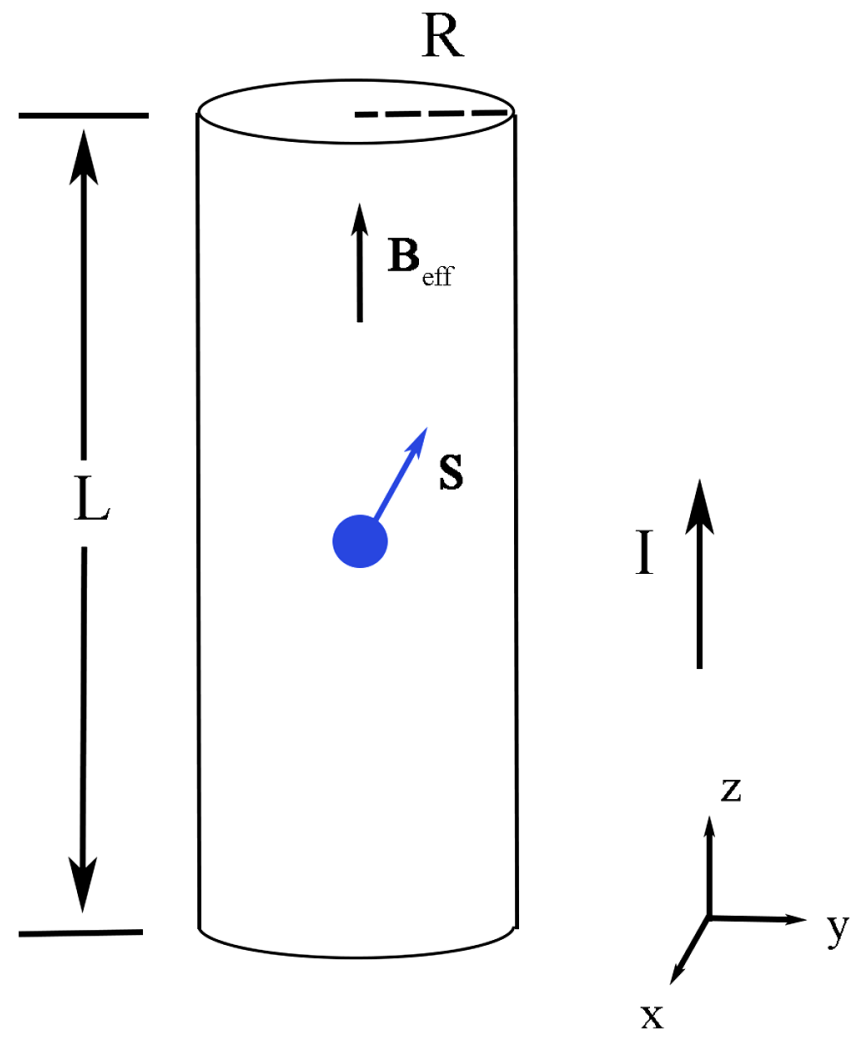

FIG. 2. An example experimental setup to illustrate the effective interaction. A cylinder-shaped conductor with length $L$ and radius $R$ is placed along $z$ axis. The conductor is hollow and an electric current flows in $+z$ direction in the surface of the conductor. Note that the magnetic field in the center of the cylinder is zero. But if axionic dark matter is present, an effective oscillating magnetic field is created in $z$ direction. Hence the spin of an electron (usually a molecule in experiments) in the center of the conductor will be affected and the spin precesses.

center of the cylinder is zero. However, axionic dark matter induces an effective magnetic field oscillating at a frequency $\omega=m_{a}$. In the center of the cylinder the effective magnetic field is,

$$
\vec{B}_{\text {eff }}(t)=-\frac{g_{a \gamma \gamma} I \vec{e}_{z}}{4 \pi} \frac{\partial a}{\partial t} \int_{-\frac{L}{2}}^{\frac{L}{2}} d z \frac{e^{i m_{a} \sqrt{z^{2}+R^{2}}}}{\sqrt{z^{2}+R^{2}}} .
$$

For ultralight axions with $m_{a} L \ll 1$ and $m_{a} R \ll 1$, the results are easily obtained,

$$
\vec{B}_{\text {eff }}(t)=-\frac{g_{a \gamma \gamma} I \vec{e}_{z}}{4 \pi} \frac{\partial a}{\partial t} \ln \frac{L+\sqrt{L^{2}+4 R^{2}}}{-L+\sqrt{L^{2}+4 R^{2}}} .
$$

If we further assume that $R \ll L$, the results become,

$$
\vec{B}_{\text {eff }}(t)=-\frac{g_{a \gamma \gamma} I \vec{e}_{z}}{2 \pi} \frac{\partial a}{\partial t} \ln \frac{L}{R} .
$$

In Fig. 3 we plot the amplitude of effective magnetic field in the center of the conductor shown in Fig. 2 for 


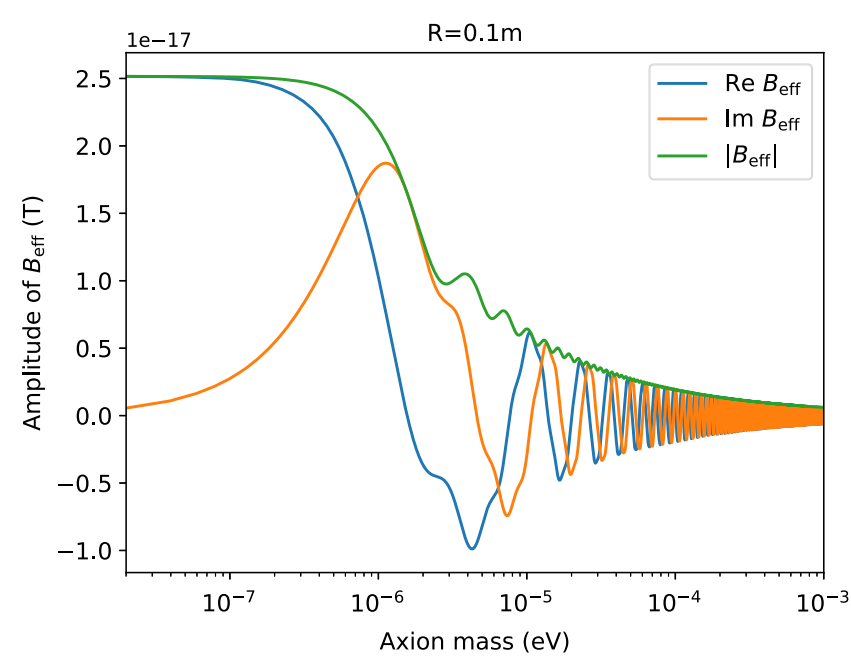

FIG. 3. Effective magnetic field obtained from Eq. (24) in the center of the cylinder-shaped conductor in Fig. 2 for different axion masses. We have fixed $L / R=10$ and $R=0.1 \mathrm{~m}$. We take $g_{\text {ary }}=10^{-12} \mathrm{GeV}^{-1}$ and the dark matter density $\rho_{\mathrm{DM}}=0.3 \mathrm{GeV} / \mathrm{cm}^{3}$. The electric current is taken as $I=$ $2 \pi R B$ with $B=10 \mathrm{~T}$. Note that the effective magnetic field oscillates periodically with frequency $\omega \simeq m_{a}$, and the green line represents its amplitude $\left|\vec{B}_{\text {eff }}\right|$. The blue and orange lines represent the real and the imaginary parts of $\vec{B}_{\text {eff }}$. In the case that the size of the conductor is smaller than the Compton wavelength of axions, the amplitude of the effective magnetic field approaches a constant.

different axion masses. We take $L / R=10, R=0.1 \mathrm{~m}$ and

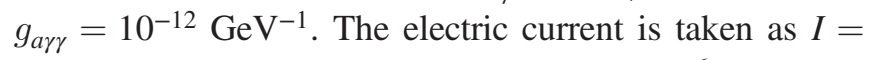
$2 \pi R B$ with $B=10 \mathrm{~T}$. Axion mass $m_{a}=10^{-6} \mathrm{eV}$ corresponds to a Compton wavelength $\lambda_{a}=1.2 \mathrm{~m}$. It is obvious that if the spatial size of the conductor is much smaller than the axion Compton wavelength, the amplitude of effective magnetic field approaches a constant value. While for a larger axion mass, the amplitude of effective magnetic field becomes smaller.

As an example, we will compare the effective interaction to the sensitivity of electron anomalous magnetic moment measurements. We adopt a magnetic field $B \sim 10 \mathrm{~T}$, and use a characteristic magnetic field $B=I /(2 \pi R)$ to estimate the effective magnetic field in Eq. (26). Note that the characteristic magnetic field is not the magnetic field in the center of the cylinder, which is zero. The effective interaction can be regarded as a modification to the electron magnetic moment in such cases, but we stress that it is intrinsically different from a magnetic moment. For an electron whose spin is aligned in $z$ direction, the effective Hamiltonian is,

$$
H_{\mathrm{eff}}(t)=\mu_{B} g_{a \gamma \gamma} B R \frac{\partial a}{\partial t} \ln \frac{L}{R}
$$

We thus find an electron effective magnetic moment,

$$
\left|\mu_{\mathrm{eff}}(t)\right|=\mu_{B} g_{a \gamma \gamma} R\left|\frac{\partial a}{\partial t}\right| \ln \frac{L}{R}
$$

Take for example $L / R=10$, we obtain,

$$
\begin{aligned}
\mu_{\mathrm{eff}}(t)= & 2.5 \times 10^{-18} \mu_{B}\left(\frac{g_{\text {ary }}}{10^{-12} \mathrm{GeV}^{-1}}\right)\left(\frac{R}{0.1 \mathrm{~m}}\right) \\
& \times\left(\frac{\rho_{\mathrm{DM}}}{0.3 \mathrm{GeV} / \mathrm{cm}^{3}}\right)^{\frac{1}{2}} \sin m_{a} t,
\end{aligned}
$$

which is roughly five orders of magnitude below the experimental sensitivity of anomalous magnetic moment measurements at present [41]. The quantity in Eq. (29) is not an electron anomalous magnetic moment, because $\mu_{\text {eff }}$ does not couple to the actual magnetic field (but couples to $B=I /(2 \pi R))$ and it depends on the experimental settings.

As an example, we consider detection of ultralight axions with $m_{a} L \ll 1$. Our calculation shows that the effects of axion-photon interaction on electron spin only appear for nonzero magnetic vector potential in Eq. (22) when considering static external fields. For many spin precession or dipole moment experiments, the electron is placed in the center of a uniform magnetic field, where the vector potential is zero. For example, if the electric current is arranged as a circle, and electrons or molecules in experiment are placed in the position which projects to the center of the circle, there will be no detectable axion effects because the magnetic vector potential is zero in that position.

We stress that the effective spin couplings induced by the axion-photon interaction should not be regarded as an electron OEDM. For example, if only a static electric field is applied, the magnetic field in Eq. (12) is zero and hence no axion-spin effective interaction for zero-velocity axions. This behavior is in contrast with usual EDM or OEDM. The result is consistent with the results in Ref. [27], but the author there incorrectly claimed that it is an electron OEDM. In fact, we find the effective interaction is not related to the electric field but related to $\nabla \times \vec{B}$. Hill [27] obtained a nonzero result for a time-varying electric field because that the time-varying electric fields are always accompanied with magnetic fields. Hill [27] obtained an effective action, ${ }^{2}$

$$
\begin{aligned}
S= & \frac{1}{2} \mu_{B} g_{a \gamma \gamma} \int d^{4} x d^{4} y a(x) F^{\mu \nu}(x) \\
& \times\left[S_{\mu \nu}^{0}(x) \delta^{4}(x-y)+\partial_{[\mu} G(x-y) \partial^{\lambda} S_{\nu] \lambda}^{0}(y)\right],
\end{aligned}
$$

where $S_{\mu \nu}^{0}=\bar{\psi} \sigma_{\mu \nu} \gamma^{5} \psi$. The first term indeed has the form of an OEDM interaction in nonrelativistic limit. But the effective action also includes the second term, which is a

\footnotetext{
${ }^{2}$ Note that we have used a slightly different definition of $g_{\text {ary }}$ from Ref. [27].
} 
nonlocal interaction. When considering a nonrelativistic electron interacting with a static electric field (no magnetic field), the second term exactly cancels the first term after integration. As we have shown, the effective axion-spin coupling exists even if the electric field is zero. Hence the point that axions induce an OEDM of the electron due to axion-photon interaction is somehow misleading.

Chu et al. [30] proposed an experiment to detect axionphoton interaction and use a constant electric field and a uniform magnetic field to detect electron OEDM. However, our calculation shows that their experiment cannot detect axion-photon interaction if we neglect the axion velocity. ${ }^{3}$ As we will discuss in Sec. III, the experiment can detect axion-electron interaction in principle, but the effect may be much weaker than their expectations.

Another interesting fact is that the result is proportional to $m_{a} a(t)$ and hence the time derivative of axion field. As we have illustrated, the axion-photon interaction satisfies axion shift symmetry, hence its physical effect is proportional to the derivative of the axion field. Thus an extra factor of $m_{a}$ appears, which suppresses the effective interaction for ultralight axions. The density of axionic dark matter is given by,

$$
\rho_{\mathrm{DM}}=\frac{1}{2} m_{a}^{2} a_{0}^{2}
$$

Hence $a_{0}$ is proportional to $1 / m_{a}$ for a fixed dark matter density. The axion induced spin interaction Hamiltonian is proportional to $a_{0}$ and hence to $1 / m_{a}$. If there were no axionshift symmetry, we would have concluded that $g_{a \gamma \gamma}$ can be strongly constrained in experiments searching for ultralight axions. Due to the axion shift symmetry, the effect of axioninteraction is proportional to time or spatial derivatives of the axion field. Hence an extra $m_{a}$ appears, canceling the $1 / m_{a}$ factor. Thus the effect on the spin precession rate is not enhanced for a smaller axion mass. However, for those axion interactions that do not obey the shift symmetry, for example axion-neutron interaction which gives a neutron OEDM, the spin precession effects are proportional to $1 / m_{a}$. Such ALP interactions are strongly constrained for ultralight axionic dark matter.

\section{B. Axion velocity effects}

The motion of the Solar system in the Milky Way results in the relative velocity of axionic dark matter as seen from the Earth. The axion velocity is $v \sim 10^{-3} c$ in terrestrial laboratories. If the axion has a nonzero velocity, we must keep the spatial derivative of the axion field in Eq. (11). The contribution to the amplitude can be obtained using the same calculation strategies,

\footnotetext{
${ }^{3}$ Velocity effects are suppressed by a factor of $v / c \sim 10^{-3}$.
}

$$
\begin{aligned}
\mathcal{M}_{p}= & \frac{i \mu_{B} g_{a \gamma \gamma}}{4 \pi} \int d^{4} x \psi^{\dagger}(x) \sigma^{k} \psi(x) a(x) \int d^{3} \vec{y} \\
& \times \frac{e^{-i \vec{p} \cdot \vec{r}}}{r} e^{i m_{a} r}\left[\vec{p}^{2} E^{k}(\vec{y})-(\vec{p} \cdot \vec{E}(\vec{y})) p^{k}\right. \\
& \left.-i p^{i} \partial_{i} E^{k}(\vec{y})+i p^{k} \partial_{i} E^{i}(\vec{y})-m_{a} p^{i} F^{k i}(\vec{y})\right],
\end{aligned}
$$

where $\vec{p}$ is the axion momentum in the laboratory frame, and we have defined $\vec{r}=\vec{x}-\vec{y}$ and $r=|\vec{x}-\vec{y}|$. We use $a(x)=a_{0} \exp (-i p \cdot x)$ to write derivatives of the axion field as $\vec{p}$ or $m_{a}$. Here we assume that the external electric and magnetic fields are static. We can write the effective Hamiltonian (including the zero-velocity part) as,

$$
H_{\mathrm{int}}=-\mu_{B} \vec{\sigma} \cdot\left(\vec{B}_{\mathrm{eff}}+\vec{B}_{\mathrm{eff}, \mathrm{p}}\right) \text {, }
$$

where $\vec{B}_{\text {eff }}$ is shown in Eq. (20) and $\vec{B}_{\text {eff,p }}$ is,

$$
\begin{aligned}
\vec{B}_{\text {eff,p }}(x)= & \frac{g_{a \gamma \gamma} a(x)}{4 \pi} \int d^{3} \vec{y} \frac{e^{-i \vec{p} \cdot \vec{r}}}{r} e^{i m_{a} r} \\
& \times\left[\vec{p}^{2} \vec{E}(\vec{y})-(\vec{p} \cdot \vec{E}(\vec{y})) \vec{p}-i(\vec{p} \cdot \nabla) \vec{E}(\vec{y})\right. \\
& \left.+i \vec{p}(\nabla \cdot \vec{E}(\vec{y}))+m_{a} \vec{p} \times \vec{B}(\vec{y})\right] .
\end{aligned}
$$

The results include three types of terms in the limit $m_{a} L \ll 1$, where $L$ is the characteristic spatial size of the external electric or magnetic field. The first two terms (after integrating over $\vec{y}$ ) are of order $p^{2} L^{2}$. The third and the fourth terms are of order $p L$. The last term is of order $m_{a} p L^{2}$. Here $m_{a} L$ is easily evaluated to be,

$$
\begin{gathered}
m_{a} L=5.07\left(\frac{m_{a}}{10^{-6} \mathrm{eV}}\right)\left(\frac{L}{\mathrm{~m}}\right) . \\
p L \approx 10^{-3} m_{a} L .
\end{gathered}
$$

For ultralight axions whose mass is smaller than about $10^{-10} \mathrm{eV}$, we have $m_{a} L \ll 1$ and $p L \ll 1$ for $L \sim 1 \mathrm{~m}$. Hence we obtain an approximate expression of the effective magnetic field for ultralight axions,

$$
\begin{aligned}
\vec{B}_{\mathrm{eff}, \mathrm{p}}= & \frac{g_{a \gamma \gamma} a(x)}{4 \pi} \int d^{3} \vec{y} \frac{1}{r} \\
& \times[-i(\vec{p} \cdot \nabla) \vec{E}(\vec{y})+i \vec{p}(\nabla \cdot \vec{E}(\vec{y}))] .
\end{aligned}
$$

We note that the velocity effect appears only for a nonuniform external electric field if $m_{a} L \ll 1$. For QCD axions with mass from $10^{-5} \mathrm{eV}$ to $10^{-2} \mathrm{eV}$, however, all terms in Eq. (34) are important. Thus for a uniform electric field, the QCD axions induced spin interactions are nonzero.

We also note that the effective magnetic field depends on the direction of axion velocity and external fields. Hence if 
such effects are detectable, they will help us determine the motion of dark matter relative to the Earth.

\section{Time-varying external fields}

We can easily extend the result to the case where the external field is time-varying. We will consider the case that the external electromagnetic fields are changing periodically in time,

$$
\vec{B}(\vec{x}, t)=\vec{B}_{0}(\vec{x}) e^{-i \omega t},
$$

where we have assumed that $\omega>0$. Here we will assume that axion velocity is zero, and can be written as $a(\vec{x}, t)=a_{0} e^{-i\left(m_{a} t+\varphi\right)} / 2+$ c.c.. We use a slightly different notation, which is necessary to obtain the correct result. As shown in Sec. II B, velocity effects are suppressed by at least a factor of $10^{-3}$, and here we neglect them for simplicity.

The amplitude can be calculated as before, and the effective interaction can be written as,

$$
H_{\mathrm{int}}=-\mu_{B} \vec{\sigma} \cdot \vec{B}_{\mathrm{eff}, \mathrm{t}},
$$

where

$$
\begin{aligned}
\vec{B}_{\mathrm{eff}, \mathrm{t}}(\vec{x}, t)= & i g_{a \gamma \gamma} m_{a} a_{0} \int d^{3} y \frac{1}{8 \pi r} \\
& \times\left[e^{i\left(\omega+m_{a}\right)(r-t)} e^{-i \varphi}-e^{i\left(\omega-m_{a}\right)(r-t)} e^{i \varphi}\right]\left(\nabla \times \vec{B}_{0}\right) .
\end{aligned}
$$

The effects are similar to the static external field case, but the effective magnetic field becomes a mixture of two frequencies $\omega+m_{a}$ and $\left|\omega-m_{a}\right|$. For ultralight axions with $m_{a} \ll \omega$, the two frequencies are almost equal. For the QCD axions and the external field frequency $\omega \sim m_{a}$, the first term in Eq. (40) is a fast oscillating term. The second term in Eq. (40) is slowly varying. Hence the first term can be neglected when considering processes like electron spin precession. However, when considering axion induced atomic transition processes, the two terms are both important.

\section{EFFECTS OF AXION-ELECTRON INTERACTION}

Axionic dark matter may also interact directly with electrons. In the following we will find out the effective electron spin interactions induced by axions.

Here we must distinguish two types of axion-electron effective interaction, namely $\mathcal{L}_{1}=-i \lambda a \bar{\psi} \gamma \gamma_{5} \psi$ and $\mathcal{L}_{2}=g_{\text {aee }}\left(\partial_{\mu} a\right) \bar{\psi} \gamma \gamma^{\mu} \gamma_{5} \psi$. These two effective Lagrangian densities are frequently used in literature. If we integrate by parts in the action and then use the Dirac equation, the two interactions seem to be equivalent if $g_{\text {aee }}=\lambda /\left(2 m_{e}\right)$.
Indeed, in the Feynman diagram if the two electron lines connected to the axion line are on shell, $\mathcal{L}_{1}$ and $\mathcal{L}_{2}$ are the same. But more generally the two interactions are different and may lead to different physical effects. Here we note that $\mathcal{L}_{1}$ does not satisfy the axion shift symmetry while $\mathcal{L}_{2}$ does. For general ALPs, we do not have reasons to impose axion shift symmetry. But for those ALPs related to a spontaneously broken global U(1) symmetry like the QCD axion, the shift symmetry should be satisfied. The most important result we obtained in the following is that the parameter $\lambda$ can be strongly constrained from spin-precession or OEDM experiments for ultralight axions while the constraints for $g_{\text {aee }}$ are much weaker. ${ }^{4}$

In Ref. [32], the interaction Lagrangian $\mathcal{L}_{1}=-i \lambda a \bar{\psi} \gamma_{5} \psi$ is used to derive the electron OEDM. Our result for the electron OEDM differs from their result by a factor of $1 / 4$, but the effective OEDM interaction Hamiltonian is the same. There could be a typo in their final result. In the following, we will consider $\mathcal{L}_{1}$ and $\mathcal{L}_{2}$ separately.

We will derive the results through field equations in the following, which is more straightforward. The same results can also be carefully obtained through Feynman diagrams. We take $\mathcal{L}_{1}=-i \lambda a \bar{\psi} \gamma_{5} \psi$ first. Field equation can be derived from the Lagrangian density,

$$
\begin{aligned}
\mathcal{L}= & -\frac{1}{4} F_{\mu \nu} F^{\mu \nu}+\frac{1}{2} \partial_{\mu} a \partial^{\mu} a-\frac{1}{2} m_{a}^{2} a^{2} \\
& +\bar{\psi}\left(i \gamma^{\mu} D_{\mu}-m_{e}\right) \psi-i \lambda a \bar{\psi} \gamma_{5} \psi .
\end{aligned}
$$

We write the four-component spinor $\psi$ as a pair of twocomponent spinors $\chi$ and $\varphi$,

$$
\psi(x)=\left(\begin{array}{c}
\chi(x) \\
\varphi(x)
\end{array}\right) e^{-i m_{e} t},
$$

and we obtain,

$$
\begin{gathered}
\left(i \partial_{0}-e A^{0}\right) \chi+\left(i \sigma^{i} \partial_{i}+e \sigma^{i} A^{i}-i \lambda a\right) \varphi=0, \\
\left(-i \sigma^{i} \partial_{i}-e \sigma^{i} A^{i}-i \lambda a\right) \chi+\left(-i \partial_{0}-2 m_{e}+e A^{0}\right) \varphi=0 .
\end{gathered}
$$

In the nonrelativistic limit we have $i \partial_{0} \chi \ll 2 m_{e} \chi, i \partial_{0} \varphi \ll$ $2 m_{e} \varphi$ and $e A^{0} \ll 2 m_{e}$. We obtain an approximate equation in the nonrelativistic limit,

$$
\begin{aligned}
i \partial_{0} \chi= & e A^{0} \chi-\left(i \sigma^{i} \partial_{i}+e \sigma^{i} A^{i}-i \lambda a\right) \frac{1}{2 m_{e}} \\
& \times\left(1-\frac{i \partial_{0}}{2 m_{e}}+\frac{e A^{0}}{2 m_{e}}\right)\left(-i \sigma^{j} \partial_{j}-e \sigma^{j} A^{j}-i \lambda a\right) \chi,
\end{aligned}
$$

\footnotetext{
${ }^{4}$ Here we regard $\lambda$ and $g_{a e e}$ independent to the axion mass.
} 
where the derivative in the bracket must act on all terms on the right. We will only pick up those terms that involve the axion field, and neglect higher order terms. The equation has the same form as the Schrödinger equation, and the term on the right hand side can be interpreted as an effective Hamilton operator acting on $\chi$. There are terms proportional to $\partial_{0} \chi$ on the right-hand side in Eq. (45). These terms either cancel each other, or only give higher order contributions to spin couplings. The electron is assumed to be static. If the axion has zero velocity, the only term that remains is,

$$
-\frac{e \lambda a}{4 m_{e}^{2}} \sigma^{i}\left(\partial_{i} A^{0}+\partial_{0} A^{i}\right) \chi=\frac{e \lambda a}{4 m_{e}^{2}} \vec{\sigma} \cdot \vec{E} \chi,
$$

where $\vec{E}$ is the electric field. This term contributes to an OEDM. The magnitude of the electron OEDM is,

$$
\begin{aligned}
d_{e, 1}= & \frac{e \lambda a}{4 m_{e}^{2}}=4.06 \times 10^{-31} e \cdot \mathrm{cm}\left(\frac{\rho_{\mathrm{DM}}}{0.3 \mathrm{GeV} / \mathrm{cm}^{3}}\right)^{\frac{1}{2}} \\
& \times\left(\frac{10^{-5} \mathrm{eV}}{m_{a}}\right)\left(\frac{\lambda}{10^{-16}}\right) \cos \left(m_{a} t\right),
\end{aligned}
$$

Note that there is no axion shift symmetry here, so the OEDM is proportional to the axion field rather than its derivatives. Thus the OEDM obtained is inversely proportional to the axion mass. If ultralight axions are dark matter, the OEDM can be very large in principle.

The oscillation period is,

$$
\frac{2 \pi}{m_{a}}=4.14 \times 10^{-10} \mathrm{~s}\left(\frac{10^{-5} \mathrm{eV}}{m_{a}}\right) .
$$

For an axion lighter than about $10^{-17} \mathrm{eV}$, the period of OEDM oscillation is large compared with typical experimental duration, and hence can be regarded as a static EDM. For ultralight axions, $\lambda$ is strongly constrained by electron EDM experiments.

If we allow a nonzero axion velocity, another term appears:

$$
-\frac{\lambda}{2 m_{e}} \sigma^{i}\left(\partial_{i} a\right) \chi
$$

This term is the same as the effective axion interaction term $g_{a e e}(\nabla a) \cdot \vec{\sigma}$, which has been studied in various literature [23] and several experiments have been designed to detect the interaction $[33,42]$. Now we must know which term dominates here. Take the axion velocity $v \sim 10^{-3} c$, we find that the OEDM term dominates if the electric field satisfies,

$$
|\vec{E}|>5.2 \times 10^{9} \mathrm{~V} / \mathrm{m}\left(\frac{m_{a}}{\mathrm{eV}}\right)
$$

This condition is easily satisfied in experiments for an axion mass smaller than about $10^{-5} \mathrm{eV}$. The result above is consistent with Ref. [32], which has used $\mathcal{L}_{1}$ to obtain the results.

We next consider the effective interaction $\mathcal{L}_{2}=$ $g_{a e e}\left(\partial_{\mu} a\right) \bar{\psi} \gamma^{\mu} \gamma_{5} \psi$. The axion shift symmetry is satisfied here. Field equations can be similarly derived,

$$
\begin{gathered}
\left(i \partial_{0}-e A^{0}+g_{a e e} \sigma^{i} \partial_{i} a\right) \chi \\
+\left(i \sigma^{i} \partial_{i}+e \sigma^{i} A^{i}+g_{a e e} \frac{\partial a}{\partial t}\right) \varphi=0 \\
\left(-i \sigma^{i} \partial_{i}-e \sigma^{i} A^{i}-g_{a e e} \frac{\partial a}{\partial t}\right) \chi \\
+\left(-i \partial_{0}-2 m_{e}+e A^{0}-g_{a e e} \sigma^{i} \partial_{i} a\right) \varphi=0,
\end{gathered}
$$

where we have used Eq. (42). In the nonrelativistic limit, the equation becomes,

$$
\begin{aligned}
i \partial_{0} \chi= & \left(e A^{0}-g_{a e e} \sigma^{m} \partial_{m} a\right) \chi \\
& -\left(i \sigma^{i} \partial_{i}+e \sigma^{i} A^{i}+g_{a e e} \frac{\partial a}{\partial t}\right) \\
& \times \frac{1}{2 m_{e}}\left(1-\frac{i \partial_{0}}{2 m_{e}}+\frac{e A^{0}}{2 m_{e}}-\frac{g_{a e e}}{2 m_{e}} \sigma^{j} \partial_{j} a\right) \\
& \times\left(-i \sigma^{k} \partial_{k}-e \sigma^{k} A^{k}-g_{a e e} \frac{\partial a}{\partial t}\right) \chi .
\end{aligned}
$$

We neglect higher order terms and only keep those involving the axion field. Here we will not consider the spatial motion of electrons, but this can be important in experiments. We will take the external magnetic field $\vec{B}=0$ in Eq. (53) first. In Eq. (45), terms proportional to $\partial_{0} \chi$ are cancelled to the lowest order. But in Eq. (53), such terms do not cancel. To lowest order, there is an extra term on the right-hand side:

$$
-\frac{i g_{\text {aee }}}{2 m_{e}^{2}} \frac{\partial a}{\partial t}\left(i \sigma^{i} \partial_{i}+e \sigma^{i} A^{i}\right) \partial_{0} \chi
$$

Hence we must include corrections of this term by iterating $\partial_{0} \chi$ using Eq. (53). This leads to many effective interaction terms. We only pick up two dominate terms here:

$$
\begin{aligned}
& -g_{a e e} \sigma^{i}\left(\partial_{i} a\right) \chi \\
& \frac{i e g_{a e e}}{4 m_{e}^{2}} \frac{\partial a}{\partial t} \vec{\sigma} \cdot \vec{E} \chi
\end{aligned}
$$

The second term contributes to an electron OEDM, 


$$
\begin{aligned}
d_{e, 2}= & \frac{e g_{a e e} m_{a} a}{4 m_{e}^{2}}=4.06 \times 10^{-42} e \cdot \mathrm{cm} \\
& \times\left(\frac{g_{a e e}}{10^{-13} \mathrm{GeV}^{-1}}\right)\left(\frac{\rho_{\mathrm{DM}}}{0.3 \mathrm{GeV} / \mathrm{cm}^{3}}\right)^{\frac{1}{2}} \cos \left(m_{a} t\right),
\end{aligned}
$$

We note that $d_{e, 2} \ll d_{e, 1}$ for typical axion parameters with $g_{\text {aee }} \sim \lambda /\left(2 m_{e}\right)$. Hence the axion shift symmetry suppresses the induced electron OEDM. The second term is larger if the electric field satisfies,

$$
|\vec{E}|>5.3 \times 10^{15} \mathrm{~V} / \mathrm{m} .
$$

In electron EDM experiments, usually $\vec{E}$ is the effective electric field in molecules. For fully polarized ThO molecule [36], the effective electric field is $78 \mathrm{GV} / \mathrm{cm}$, which is still much smaller than $5.3 \times 10^{15} \mathrm{~V} / \mathrm{m}$ in Eq. (58). Hence the first term dominates in laboratory experiments.

In the following we will estimate the constraints on $\lambda$ and $g_{\text {aee }}$ imposed by current electron EDM experiments. This should be regarded as rough constraints and can be improved if we carefully adjust the duration of "time blocks" [35,36], within which we measure the spin precession of molecules and average over these measurements. In Ref. [36] a block lasts for $T=60 \mathrm{~s}$, which roughly means that we can only constrain ultralight axions with mass $m_{a} \lesssim 1 / T$. For a higher mass the EDM effects may be averaged out. We again take $\rho_{\mathrm{DM}}=0.3 \mathrm{GeV} / \mathrm{cm}^{3}$ here. Using the electron static EDM constraint [36] $d_{e}<1.1 \times 10^{-29} e \cdot \mathrm{cm}$, the constraint for $\lambda$ is,

$\lambda<2.71 \times 10^{-32}\left(\frac{m_{a}}{10^{-22} \mathrm{eV}}\right), \quad$ for $m_{a} \lesssim 10^{-17} \mathrm{eV}$.

The constraint for $g_{a e e}$ is,

$$
g_{a e e}<0.27 \mathrm{GeV}^{-1}, \quad \text { for } m_{a} \lesssim 10^{-17} \mathrm{eV} .
$$

It is obvious that the constraint for $g_{\text {aee }}$ is very weak compared with stellar cooling constraint, $g_{\text {aee }}<$ $2.8 \times 10^{-13} \mathrm{GeV}^{-1}$ [43]. Hence for usual axions originated from a spontaneously broken $\mathrm{U}(1)$ symmetry, electron static EDM does not give a substantial constraint. The interaction $\mathcal{L}_{1}=-i \lambda a \bar{\psi} \gamma_{5} \psi$ is not forbidden by symmetries for general ALPs, in which case $\lambda$ is strongly constrained from static EDM experiments for ultralight ALPs that compose the dark matter.

The experiments in Refs. [30,31] were designed to detect electron OEDM. As we have shown, axion-photon interaction does not contribute to an OEDM but axion-electron interaction contributes. The experiment can constrain $\lambda$, but it is difficult to give a substantial constraint for $g_{a e e}$ when considering OEDM interaction. This means that a large class of ALPs that satisfies the approximate shift symmetry is hard to detect in their experiment proposals. In contrast to the electron OEDM, the nucleon OEDM is much more likely to detect because the corresponding interaction does not satisfy the shift symmetry.

If we allow for a nonzero magnetic field, an additional effective interaction term appears:

$$
-\frac{e g_{a e e}}{2 m_{e}^{2}}(\nabla a) \cdot \vec{B} \chi
$$

This term is unrelated to the electron spin, and does not appear in the results of $\mathcal{L}_{1}$. It contributes an oscillating magnetic moment,

$$
\vec{\mu}=\frac{e g_{\text {aee }}}{2 m_{e}^{2}}(\nabla a)
$$

This term is smaller than the OEDM interaction in Eq. (56) because of the suppression of axion velocity $v \sim 10^{-3}$. The magnitude of the oscillating magnetic moment is,

$$
\begin{aligned}
\mu= & 4.2 \times 10^{-34} \mu_{B}\left(\frac{g_{\text {aee }}}{10^{-13} \mathrm{GeV}^{-1}}\right) \\
& \times\left(\frac{\rho_{\mathrm{DM}}}{0.3 \mathrm{GeV} / \mathrm{cm}^{3}}\right)^{\frac{1}{2}}\left(\frac{v}{10^{-3}}\right) \sin \left(m_{a} t\right),
\end{aligned}
$$

where the spatial dependence of the axion field contributes an unimportant phase for a static electron, which we neglect here. Such a small oscillating magnetic moment is not observable in experiments for the near future.

\section{EFFECTS OF AXION-NEUTRON INTERACTION}

We finally briefly discuss axionic dark matter induced effective interactions of the neutron spin, which is similar to the electron case. The biggest difference is that there is an extra term that contributes to the neutron OEDM [23] arising from the coupling $\left(a / f_{a}\right) G \tilde{G}$, where $G$ is the QCD field strength. Axion neutron interactions include,

$$
\begin{aligned}
\mathcal{L}_{n, 1} & =g_{\text {ann }}\left(\partial_{\mu} a\right) \bar{\psi} \gamma^{\mu} \gamma_{5} \psi, \\
\mathcal{L}_{n, 2} & =-\frac{i}{2} g_{d} a \bar{\psi} \sigma_{\mu \nu} \gamma_{5} \psi F^{\mu \nu} .
\end{aligned}
$$

The first term has the same form as the axion-electron interaction, while the second term contributes to an OEDM directly. In the nonrelativistic limit, the second term becomes,

$$
g_{d} a \chi^{\dagger}(x) \vec{\sigma} \chi(x) \cdot \vec{E}(x),
$$

where $\chi(x)$ is defined in Eq. (42). This term contributes to a neutron OEDM, 


$$
\begin{aligned}
d_{n}= & g_{d} a=1.40 \times 10^{-30} e \cdot \mathrm{cm}\left(\frac{g_{d}}{10^{-10} \mathrm{GeV}^{-2}}\right) \\
& \times\left(\frac{m_{a}}{10^{-5} \mathrm{eV}}\right)^{-1}\left(\frac{\rho_{\mathrm{DM}}}{0.3 \mathrm{GeV} / \mathrm{cm}^{3}}\right)^{\frac{1}{2}} \cos \left(m_{a} t\right) .
\end{aligned}
$$

The axion induced nucleon OEDM has been extensively studied before, and many experiments are designed to detect it $[24,25]$. We write it here for completeness. We stress that the axion-nucleon interaction $\mathcal{L}_{n, 2}$ does not satisfy the axion shift symmetry due to instanton effects, and hence the neutron OEDM is proportional to $1 / m_{a}$ for a fixed coupling constant $g_{d} .{ }^{5}$ The constraint of $g_{d}$ from current static EDM experiments has been shown in Ref. [44].

Besides, neutron has a magnetic moment $\mu_{n}=-1.042 \times$ $10^{-3} \mu_{B}$ [41]. Hence axion-photon interaction also introduces a coupling involving the neutron spin. But because the magnetic moment of neutron is about $10^{-3}$ smaller than the electron, the effect becomes difficult to detect. The reasoning is the same as that in Sec. II with a replacement $\mu_{B} \rightarrow \mu_{n}$.

\section{DISCUSSION}

If axions are the primary components of the dark matter, axion-spin effective interactions will be introduced. These interactions may manifest themselves in spin precession experiments or OEDM searches. Future detectors with improved sensitivities may be able to detect axions via these kinds of experiments.

We stress that axion-photon interaction effects on the electron spin is not the same as an electron OEDM. The effective interaction is nonzero even if the external electric field does not exist. The effect can be understood as the electron spin interacting with an effective time-varying magnetic field, whose frequency equals to the axion mass. The effective time-varying magnetic field is independent of the electron and can be derived through field equations. Hence such effects also appear for a macroscopic magnetic moment, whose size is smaller than the Compton wavelength of the axion. To detect the effective magnetic field induced by axions, spin precession experiments must be carefully arranged. For example, to detect ultralight axions, the electron must be placed where the vector potential is nonzero.

\footnotetext{
${ }^{5}$ We regard $g_{d}$ as an independent parameter here, not necessarily related to $m_{a}$.
}

When we discuss axion-photon interaction effects, we have assumed a coherent axion background field, which can be written as $a(x)=a_{0} e^{-i p \cdot x}$. However, if the scale of the external field is larger than the coherence length $L_{0} \sim 10^{3} / m_{a}$, the results in Sec. II do not apply because the axion field may have different phase in different regions. In general, it is still possible that ALPs form a condensate and have a much larger coherent length than $L_{0}$ [45].

For the axion-electron interaction, axion introduces an electron OEDM, whose magnitude depends on the form of ALP-electron interaction. Specifically, if the axion interaction obeys the shift symmetry as usual, the electron OEDM is not enhanced for a very small axion mass. If the axion interaction does not obey the shift symmetry, the electron OEDM is large for ultralight axions. For an ultralight axion as dark matter with a mass lighter than $10^{-17} \mathrm{eV}$, the oscillation period of OEDM is large and it can be regarded as a static EDM in electron spin precession experiments. Thus such interaction parameters including $\lambda$ and $g_{d}$ are strongly constrained by current EDM experiments for ultralight axionic dark matter.

It is possible that the dark matter density near the Earth or the Sun is much larger than the local dark matter density $\rho_{\mathrm{DM}}=0.3 \mathrm{GeV} / \mathrm{cm}^{3}$ [46]. For example, ALPs may form miniclusters or dilute axion stars $[47,48]$. If the Earth passes through such high density region, the axion induced spin interactions will be easier to detect. Another possibility is that some ALPs are trapped in the gravitational potential of the Earth and the Sun, which may increase the dark matter density by a factor of $10^{4}$ [49]. This may enhance the axion induced spin interaction by a factor $10^{2}$. But these scenarios have large uncertainties and are still controversial.

\section{ACKNOWLEDGMENTS}

We thank Li-Xin Li and Junwu Huang for helpful discussions. Z. W. was supported by the National Natural Science Foundation of China (Grant No. 11973014). L. S. was supported by the Young Elite Scientists Sponsorship Program by the China Association for Science and Technology (Grant No. 2018QNRC001), the National Natural Science Foundation of China (Grants No. 11975027 and No. 11991053), the National SKA Program of China (Grant No. 2020SKA0120300), and the Max Planck Partner Group Program funded by the Max Planck Society. 
[1] G. Bertone, D. Hooper, and J. Silk, Particle dark matter: Evidence, candidates and constraints, Phys. Rep. 405, 279 (2005).

[2] E. J. Copeland, M. Sami, and S. Tsujikawa, Dynamics of dark energy, Int. J. Mod. Phys. D 15, 1753 (2006).

[3] G. Jungman, M. Kamionkowski, and K. Griest, Supersymmetric dark matter, Phys. Rep. 267, 195 (1996).

[4] G. Arcadi, M. Dutra, P. Ghosh, M. Lindner, Y. Mambrini, M. Pierre, S. Profumo, and F. S. Queiroz, The waning of the WIMP? A review of models, searches, and constraints, Eur. Phys. J. C 78, 203 (2018).

[5] R. D. Peccei and H. R. Quinn, $C P$ Conservation in the Presence of Pseudoparticles, Phys. Rev. Lett. 38, 1440 (1977).

[6] S. Weinberg, A New Light Boson?, Phys. Rev. Lett. 40, 223 (1978).

[7] G. G. di Cortona, E. Hardy, J. P. Vega, and G. Villadoro, The QCD axion, precisely, J. High Energy Phys. 2016 (2016) 37.

[8] P. Svrcek and E. Witten, Axions in string theory, J. High Energy Phys. 06 (2006) 051.

[9] D. J. E. Marsh, Axion cosmology, Phys. Rep. 643, 1 (2016).

[10] J. E. Kim, QCD axion and quintessential axion, Part. Phys. Stand. Model 92, 665 (2004).

[11] W. Hu, R. Barkana, and A. Gruzinov, Fuzzy Cold Dark Matter: The Wave Properties of Ultralight Particles, Phys. Rev. Lett. 85, 1158 (2000).

[12] A. de Angelis, M. Roncadelli, and O. Mansutti, Evidence for a new light spin-zero boson from cosmological gammaray propagation?, Phys. Rev. D 76, 121301(R) (2007).

[13] J. Preskill, M. B. Wise, and F. Wilczek, Cosmology of the invisible axion, Phys. Lett. 120B, 127 (1983).

[14] L. F. Abbott and P. Sikivie, A cosmological bound on the invisible axion, Phys. Lett. 120B, 133 (1983).

[15] M. Dine and W. Fischler, The not-so-harmless axion, Phys. Lett. 120B, 137 (1983).

[16] M. Giannotti, I. Irastorza, J. Redondo, and A. Ringwald, Cool WISPs for stellar cooling excesses, J. Cosmol. Astropart. Phys. 05 (2016) 057.

[17] D. H. Weinberg, J. S. Bullock, F. Governato, R. Kuzio de Naray, and A. H. G. Peter, Cold dark matter: Controversies on small scales, Proc. Natl. Acad. Sci. U.S.A. 112, 12249 (2015).

[18] N. Du, N. Force, R. Khatiwada, E. Lentz, R. Ottens, L. J. Rosenberg et al., Search for Invisible Axion Dark Matter with the Axion Dark Matter Experiment, Phys. Rev. Lett. 120, 151301 (2018).

[19] T. Braine, R. Cervantes, N. Crisosto, N. Du, S. Kimes, L. J. Rosenberg et al., Extended Search for the Invisible Axion with the Axion Dark Matter Experiment, Phys. Rev. Lett. 124, 101303 (2020).

[20] R. Ballou, G. Deferne, M. Finger, M. Finger, L. Flekova, J. Hosek et al., New exclusion limits on scalar and pseudoscalar axionlike particles from light shining through a wall, Phys. Rev. D 92, 092002 (2015).

[21] K. van Bibber, P. M. McLntyre, D. E. Morris, and G. G. Raffelt, Design for a practical laboratory detector for solar axions, Phys. Rev. D 39, 2089 (1989).

[22] E. Aprile et al., Excess electronic recoil events in XENON1T, Phys. Rev. D 102, 072004 (2020).
[23] P. W. Graham and S. Rajendran, New observables for direct detection of axion dark matter, Phys. Rev. D 88, 035023 (2013).

[24] A. Garcon et al., The cosmic axion spin precession experiment (CASPEr): A dark-matter search with nuclear magnetic resonance, Quantum Sci. Technol. 3, 014008 (2018).

[25] S. P. Chang, H. Selçuk, O. Kim, S. Lee, S. Park, and Y. K. Semertzidis, Axionlike dark matter search using the storage ring EDM method, Phys. Rev. D 99, 083002 (2019).

[26] C. T. Hill, Axion induced oscillating electric dipole moments, Phys. Rev. D 91, 111702 (2015).

[27] C. T. Hill, Axion induced oscillating electric dipole moment of the electron, Phys. Rev. D 93, 025007 (2016).

[28] C. T. Hill, Reply to Comment on 'axion induced oscillating electric dipole moments', Phys. Rev. D 95, 058702 (2017).

[29] C. T. Hill, Theorem: A static magnetic N-pole becomes an oscillating electric N-pole in a cosmic axion field, arXiv: 1606.04957.

[30] P. H. Chu, Y. J. Kim, and I. Savukov, Search for an axioninduced oscillating electric dipole moment for electrons using atomic magnetometers, Phys. Rev. D 99, 075031 (2019).

[31] P. H. Chu, Y. J. Kim, and I. Savukov, Comment on Search for an axion-induced oscillating electric dipole moment for electrons using atomic magnetometers, arXiv:1904.10543.

[32] S. Alexander and R. Sims, Detecting axions via induced electron spin precession, Phys. Rev. D 98, 015011 (2018).

[33] Y. V. Stadnik and V. V. Flambaum, Axion-induced effects in atoms, molecules, and nuclei: Parity nonconservation, anapole moments, electric dipole moments, and spin-gravity and spin-axion momentum couplings, Phys. Rev. D 89, 043522 (2014).

[34] V. V. Flambaum and H. B. Tran Tan, Oscillating nuclear electric dipole moment induced by axion dark matter produces atomic and molecular electric dipole moments and nuclear spin rotation, Phys. Rev. D 100, 111301(R) (2019).

[35] J. Baron et al., Order of magnitude smaller limit on the electric dipole moment of the electron, Science 343, 269 (2014).

[36] V. Andreev et al., Improved limit on the electric dipole moment of the electron, Nature (London) 562, 355 (2018).

[37] A. B. Balakin and V. A. Popov, Spin-axion coupling, Phys. Rev. D 92, 105025 (2015).

[38] J. E. Moody and F. Wilczek, New macroscopic forces?, Phys. Rev. D 30, 130 (1984).

[39] J. Ouellet and Z. Bogorad, Solutions to axion electrodynamics in various geometries, Phys. Rev. D 99, 055010 (2019).

[40] M. Beutter, A. Pargner, T. Schwetz, and E. Todarello, Axion-electrodynamics: A quantum field calculation, J. Cosmol. Astropart. Phys. 02 (2019) 026.

[41] P. A. Zyla et al., Review of particle physics, Prog. Theor. Exp. Phys. 2020, 083 C01 (2020).

[42] P. W. Graham, D. E. Kaplan, J. Mardon, S. Rajendran, W. A. Terrano, L. Trahms, and T. Wilkason, Spin precession experiments for light axionic Dark Matter, Phys. Rev. D 97, 055006 (2018). 
[43] M. M. Miller Bertolami, B. E. Melendez, L. G. Althaus, and J. Isern, Revisiting the axion bounds from the Galactic white dwarf luminosity function, J. Cosmol. Astropart. Phys. 10 (2014) 069.

[44] N. J. Ayres, Hunting for axionlike dark matter by searching for an oscillating neutron electric dipole moment, arXiv: 1805.10252.

[45] P. Sikivie and Q. Yang, Bose-Einstein Condensation of Dark Matter Axions, Phys. Rev. Lett. 103, 111301 (2009).
[46] A. Banerjee et al., Searching for Earth/Solar axion halos, J. High Energy Phys. 2020 (2020) 004.

[47] E. W. Kolb and I. I. Tkachev, Axion Miniclusters and Bose Stars, Phys. Rev. Lett. 71, 3051 (1993).

[48] Z. Wang, L. Shao, and L.-X. Li, Resonant instability of axionic dark matter clumps, J. Cosmol. Astropart. Phys. 07 (2020) 038.

[49] X. Xu and E. R. Siegel, Dark matter in the solar system, arXiv:0806.3767. 